\section{Caracterização das epidemias de malária nos municípios da Amazônia Brasileira em 2010}

\author{
Characteristics of malaria epidemics in the \\ municipalities of the Brazilian Amazon, 2010
}

\section{Caracterización de las epidemias de malaria en los municipios de la Amazonia brasileña en 2010}

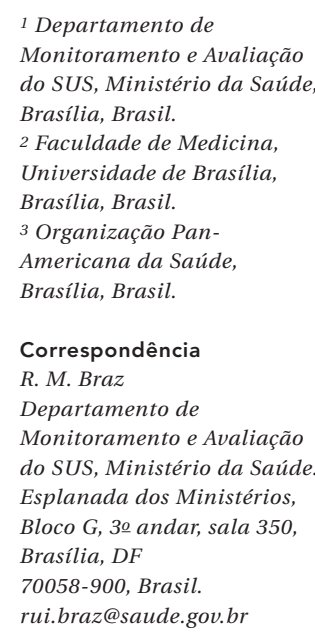

\begin{abstract}
Malaria epidemics occur annually in various municipalities (counties) in the Brazilian Amazon. However, health services do not systematically adopt tools to detect and promptly control these events. This article aimed to characterize malaria epidemics in the Brazilian Amazon Region based on their duration, the Plasmodium species involved, and the population's degree of vulnerability. An automatic malaria incidence monitoring system based on quartiles was assessed for prompt identification of malaria epidemics. In 2010, epidemics were identified in 338 (41.9\%) of the counties in the Brazilian Amazon. P. falciparum and P. vivax epidemics were detected, both singly and in combination. Epidemics lasted from 1 to 4 months in 58.3\% of the counties, 5 to 8 months in 34.5\%, and 9 to 12 months in $17.4 \%$. Systematic monitoring of malaria incidence could contribute to early detection of epidemics and improve the effectiveness of control measures.
\end{abstract}

Malaria; Plasmodium; Epidemics
Rui Moreira Braz 1,2

Elisabeth Carmen Duarte 2,3

Pedro Luiz Tauil 2

Epidemias de malária ocorrem anualmente nos municípios da Região Amazônica, Brasil, no entanto os serviços de saúde não adotam, de maneira sistemática, instrumentos para detecção e contenção oportunas desses eventos. O objetivo foi caracterizar as epidemias de malária na região segundo duração, espécie de Plasmodium e vulnerabilidade das populações. Foi avaliado um sistema de monitoramento automatizado da incidência da malária, com base no diagrama de controle segundo quartis, para identificar as epidemias da doença. Em 2010, ocorreram epidemias em 338 (41,9\%) municípios da região. Houve epidemias por $\mathrm{P}$. falciparum $e$ por $\mathrm{P}$. vivax, separadamente, e também por ambas as espécies. Epidemias com duração de um a quatro meses ocorreram em 58,3\% dos municípios epidêmicos; de cinco a oito meses, em 24,3\%; e de nove a 12 meses, em 17,4\%. O monitoramento automatizado da variação da incidência da malária poderá contribuir para detecção precoce das epidemias e melhorar o seu controle oportuno.

Malária; Plasmodium; Epidemia 


\section{Introdução}

A malária permanece como sério problema de saúde pública no Brasil. Dados da Organização Mundial da Saúde (OMS) indicam que o país é responsável por $55 \%$ dos casos da doença notificados nas Américas 1. Em 2010, no Brasil, foram notificados 334 mil casos, sendo 99,6\% na Região Amazônica. Avanços na redução da mortalidade e da internação hospitalar por malária foram alcançados, porém a transmissão continua elevada 2 . O número de casos incidentes da doença em 2010 foi próximo daquele em 2002 (348.259 casos), mostrando que, em quase uma década, não houve redução efetiva desse indicador. Além disso, epidemias de malária são registradas anualmente, distribuídas por diversos municípios da região. Em 2003, foi implantado um sistema de informação para vigilância epidemiológica da malária (Sivep-Malária), que permite monitorar a ocorrência de casos e avaliar a cobertura de diagnóstico e tratamento ${ }^{3}$. Contudo, não está disponível e/ou não é prática rotineira o uso de mecanismo automatizado para detecção oportuna de epidemias, conforme metodologia proposta por organismos internacionais 4 . Esse método deve ser simples, para ser aplicado em locais com baixos níveis de tecnologia; reprodutível, para indicar níveis anormais de incidência da malária em diferentes contextos; e válido (sensível), para alertar, precoce e oportunamente, as verdadeiras variações acima do esperado, visando ao controle efetivo de epidemias 5 .

Estudos realizados na África indicam que as epidemias são causadas por condições anormais do tempo, combinadas com aumento de resistência às drogas, mobilidade populacional, comoção civil e redução ou inadequação das ações de controle 6 . Na Amazônia Brasileira, a incidência da malária está associada às interações entre a forma de uso da terra, o comportamento humano, os elementos físicos e biológicos do meio natural e as transformações nele causadas pela intervenção humana 7, como, por exemplo, colonização agrícola, pecuária, extrativismo vegetal e mineral ${ }^{8}$, além da construção de estradas e de usinas hidrelétricas. No contexto brasileiro, deve ser fortemente destacada a determinação social da malária; portanto, merecem atenção os fatores socioeconômicos que, em certa medida, modulam o autocuidado, a exposição aos fatores de risco, a identificação da doença como problema de saúde pública, as condições para a adoção de meios de prevenção e a adesão ao tratamento. Em adição, os determinantes políticos interagem nessa complexa rede de causalidade, com especial destaque para os diferenciais de gestão em saúde pública nos três níveis de go- verno - municipal, estadual e federal - ao longo do tempo.

Para o controle da malária, além da identificação e intervenção nos seus determinantes, é necessário conhecer as variações sazonais, cíclicas e históricas da doença em cada localidade, assim como identificar precocemente suas mudanças não esperadas - o fenômeno epidêmico -, descrevendo onde e quando ele ocorre e quais as áreas com populações mais afetadas. Considerando esse contexto, o diagrama de controle por quartis tem sido visto como uma ferramenta simples, porém importante, cujo uso deve ser operacionalmente facilitado. Assim, foi desenvolvida uma ferramenta eletrônica para automação do diagrama de controle, que permitiu caracterizar as epidemias de malária na região. Este método, recomendado pela OMS e implantado em países africanos ${ }^{9}$, foi testado no Brasil e indicado para área endêmica 10 , entretanto sua implantação ainda não foi concluída. O objetivo do presente estudo foi caracterizar as epidemias de malária ocorridas na Amazônia Brasileira, em 2010, segundo as espécies de Plasmodium envolvidas, e ainda descrever a distribuição dos municípios epidêmicos por estados, por duração das epidemias e por áreas com populações de características especiais.

\section{Métodos}

\section{Área e municípios de estudo}

O presente estudo tem como unidade de análise os 807 municípios dos estados da Amazônia Brasileira. Essa região é composta pelos Estados do Acre, Amapá, Amazonas, Maranhão, Mato Grosso, Pará, Rondônia, Roraima e Tocantins. Entre os 807 municípios destes nove estados, 52 fazem fronteira com sete países: Bolívia, Colômbia, Guiana, Guiana Francesa, Peru, Suriname e Venezuela. A população total dessa região era de 25.469.352 habitantes em 2010.

\section{Sistema de Monitoramento de Casos de} Malária na Amazônia Brasileira (SIMAM)

O SIMAM foi desenvolvido a partir da elaboração de um algoritmo que permitiu a automação do sistema. Foram utilizados códigos de linguagem do programa Epi Info (Centers for Disease Control and Prevention, Atlanta, Estados Unidos). O sistema processou casos individuais de malária, registrados no Sivep-Malária, referentes aos anos de 2003 a 2010. Foi identificado o terceiro quartil como limite superior do diagrama de controle, para cada um dos municípios estudados. Esta in- 
vestigação utilizou apenas um dos módulos do SIMAM, o qual permitiu identificar os municípios com epidemias de malária segundo diversas variáveis (Figura 1). Para construção do limite superior do diagrama de controle foram usados dados mensais de casos novos de malária dos anos de 2003 a 2009, excluindo-se as duas maiores incidências de cada mês, conforme método utilizado em estudo realizado em Cruzeiro do Sul (Acre) 11. O ano definido para monitoramento foi 2010. Foram excluídas das análises as lâminas de verificação de cura. De acordo com o Programa Nacional de Controle da Malária, as lâminas de verificação de cura são classificadas como exames de microscopia realizados durante e após tratamento recente, em pacientes previamente diagnosticados para $P$. vivax ou para $P$. falciparum, que foram tratados para $P$. vivax dentro dos últimos 60 dias do diagnóstico atual, ou para $P$. falciparum dentro dos últimos 40 dias do diagnóstico atual.

Adotou-se o conceito de epidemia como sendo a ocorrência de casos acima do que normalmente é esperado, de acordo com a OMS 12 e Medronho \& Perez 13. Toda incidência mensal, acima de um caso, que ultrapassou o limite superior do diagrama de controle foi considerada uma epidemia (mês epidêmico).

\section{Análise de dados}

Foram analisadas as variáveis relativas às áreas com populações de características especiais, conforme registradas no Sivep-Malária: assentamentos, áreas indígenas e garimpos. O sistema Sivep-Malária inclui, entre os assentamentos, as localidades categorizadas como acampamento, gleba, projeto de assentamento dirigido e sua

\section{Figura 1}

Fluxo de funcionamento do Sistema de Monitoramento de Casos de Malária na Amazônia Brasileira (SIMAM), destacando o módulo de relatórios dos municípios com epidemias.

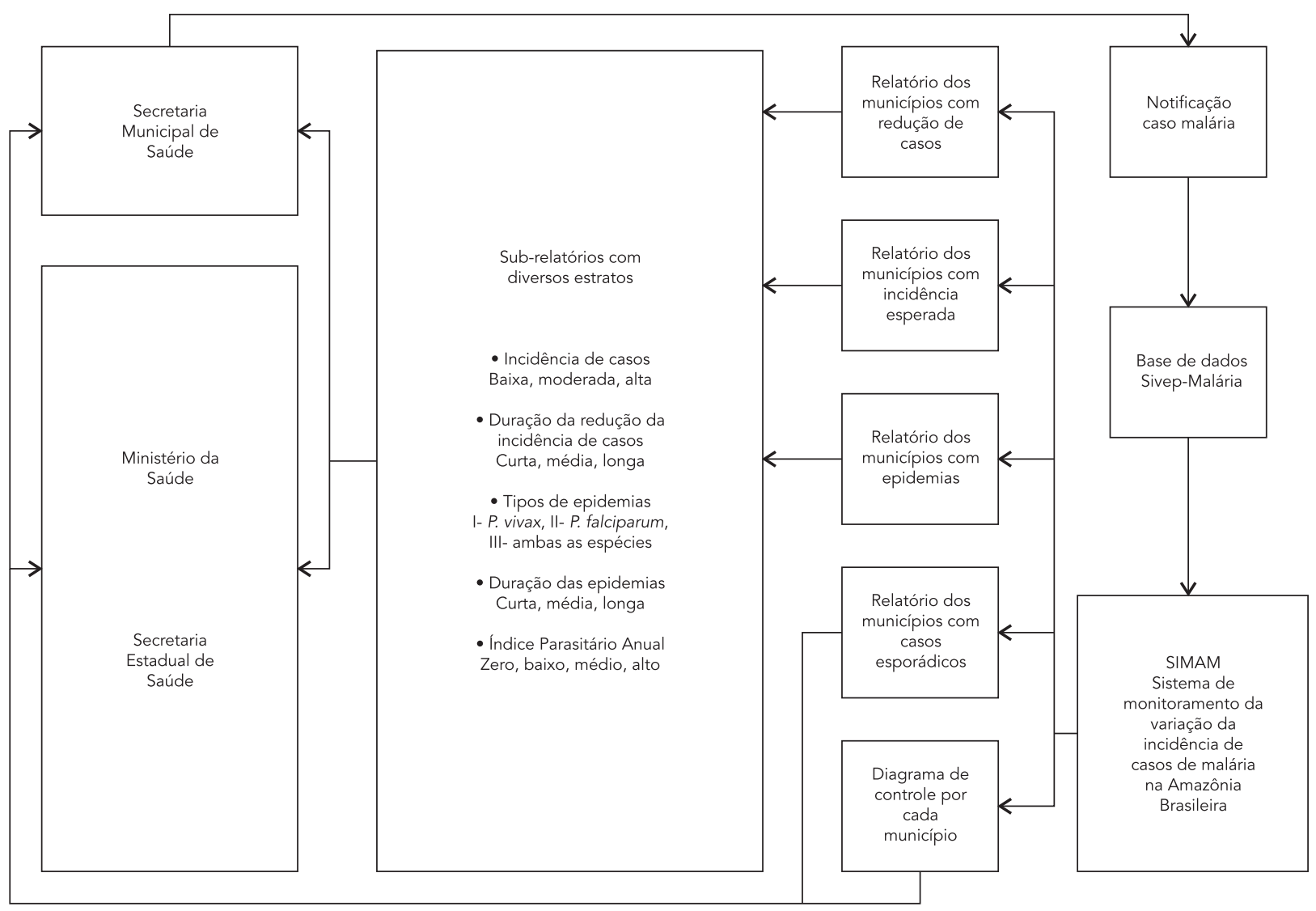


área de abrangência. Áreas indígenas incluem as malocas e aldeias. Os garimpos incluem somente esta categoria de localidade. Em relação aos municípios de fronteira, estes foram identificados no Sistema Integrado de Saúde das Fronteiras SIS-Fronteira (Secretaria de Assistência à Saúde, Ministério da Saúde. Sistema Integrado de Saúde das Fronteiras (SIS-Fronteira). http://portal. saude.gov.br/portal/saude/Gestor/visualizar_ texto.cfm? idtxt $=25617$, acessado em $02 / \mathrm{Nov} /$ 2011). A associação entre a presença de áreas com características especiais e a ocorrência de epidemias foi verificada utilizando-se o teste do $\chi^{2}$ para g.l. $=1$ e $\alpha=0,05$. A duração das epidemias foi classificada em: de curta duração (um a quatro meses epidêmicos durante o ano); de média duração (cinco a oito meses); e de longa duração (nove meses ou mais). As epidemias produzidas pelas espécies parasitárias foram categorizadas em: tipo I, produzidas pelo P. vivax; tipo II, produzidas pelo P. falciparum; e tipo III, produzidas por ambas as espécies.

\section{Considerações éticas}

O projeto foi aprovado pelo Comitê de Ética em Pesquisa da Faculdade de Medicina da Universidade de Brasília, conforme parecer no CEP-FM049/2010. A utilização dos dados foi autorizada pela Secretaria de Vigilância em Saúde do Ministério da Saúde.

\section{Resultados}

Em 2010, dos 807 municípios dos estados da Amazônia Brasileira, 543 (67,3\%) notificaram 333.398 casos de malária. Casos autóctones foram registrados por 482 (59,7\%) municípios, e epidemias ocorreram em 338 (41,9\%) deles. Em 325 (40,3\%), não houve transmissão da doença, e, em 151 destes (18,7\% da região), não ocorreu autoctonia nos últimos cinco anos. Em 63 (7,8\%) municípios, registrou-se somente um caso da doença em 2010, e nenhum caso foi notificado nos sete anos anteriores; estes municípios não foram considerados epidêmicos. As infecções por P. falciparum representaram $14,1 \%$ do total de casos notificados na região, incluindo as infecções mistas (P. falciparum + P. vivax). As infecções por $P$. malariae corresponderam a $0,05 \%$; as demais foram por P. vivax.

\section{Epidemias por espécies de Plasmodium}

Os 338 municípios epidêmicos foram distribuídos de acordo com a ocorrência dos três tipos de epidemias: tipo I, 3,3\%; tipo II, 5,6\%; tipo III,
$91,1 \%$. Ressalta-se que, nos casos das epidemias tipo III, apesar do envolvimento das duas espécies de Plasmodium (P. vivax e P. falciparum), na maioria dos municípios, prevaleceu sempre o P. vivax, em proporção superior a $85 \%$. Os Estados de Rondônia e Amapá concentraram maior número de municípios com epidemia tipo I. No Pará e Amazonas, os municípios com epidemias tipo II foram mais frequentes. A maioria dos municípios com epidemias tipo III situou-se nos Estados do Pará e Maranhão. No Maranhão e em Roraima, não houve epidemia do tipo I, e, em Tocantins, não ocorreu epidemia do tipo II. Dos 30 municípios com epidemias por apenas uma espécie de Plasmodium (tipos I e II), prevaleceram aquelas produzidas pelo $P$. falciparum $(63,3 \%)$ (Tabela 1$)$.

\section{Duração das epidemias}

Dos 338 municípios epidêmicos, em 58,3\%, as epidemias foram de curta duração; em $24,3 \%$, de média duração; e, em $17,4 \%$, de longa duração (Tabela 2). As epidemias de curta duração prevaleceram nos três grupos de municípios, conforme os tipos de epidemias. Quando analisadas as epidemias por tipos I, II e III, verificou-se que aquelas produzidas pelo $P$ falciparum, tipo II, apresentaram maior percentual de municípios com epidemias de longa duração $(25,6 \%)$, do que aquelas produzidas pelo $P$. vivax (19\%) e por ambas as espécies (19,3\%). Os municípios com epidemias de curta duração foram mais frequentes no Maranhão $(26,9 \%)$, no Pará $(27,4 \%)$ e em Mato Grosso (14,7\%). No Estado de Tocantins, as epidemias foram todas de curta duração. Encontrou-se maior frequência de municípios com epidemias de média duração no Pará $(32,9 \%)$ e no Maranhão (20,7\%). Os municípios com epidemias de longa duração concentraram-se nos Estados do Pará (50,8\%) e no Amazonas (18,6\%).

Em alguns municípios, as epidemias iniciaram em anos anteriores a 2010 e apresentaram mais de 12 meses epidêmicos. Esse foi o caso de Espigão D'Oeste (Rondônia), cuja epidemia persistiu desde 2008, totalizando 38 meses epidêmicos. Situações mais preocupantes foram detectadas em Santa Cruz do Arari (Pará), onde a epidemia iniciou em 2007, contabilizando 42 meses epidêmicos, e em Goianésia do Pará (Pará), com duração de 46 meses epidêmicos, até o final de 2010 (Figura 2).

\section{Epidemias em municípios com áreas de populações com características especiais}

Verificou-se a existência de 477 municípios com áreas especiais. Destes, 218 tiveram transmissão 
Tabela 1

Distribuição dos municípios por tipos de epidemias e por estados. Amazônia Brasileira, 2010.

\begin{tabular}{|c|c|c|c|c|c|c|c|c|c|c|}
\hline \multirow[t]{3}{*}{ Tipos de epidemias } & \multicolumn{9}{|c|}{ Número de municípios epidêmicos por estados } & \multirow[t]{3}{*}{ Total } \\
\hline & Acre & Amazonas & Amapá & Maranhão & Mato & Pará & Rondônia & Roraima & Tocantins & \\
\hline & \multicolumn{9}{|c|}{ Grosso } & \\
\hline Tipo I (Plasmodium vivax) & 1 & 2 & 2 & 0 & 1 & 1 & 3 & 0 & 1 & 11 \\
\hline Tipo II (Plasmodium & 2 & 4 & 1 & 1 & 2 & 6 & 2 & 1 & 0 & 19 \\
\hline \multicolumn{11}{|l|}{ falciparum) } \\
\hline Tipo III (ambas as espécies) & 11 & 32 & 12 & 72 & 34 & 104 & 26 & 14 & 3 & 308 \\
\hline Total & 14 & 38 & 15 & 73 & 37 & 111 & 31 & 15 & 4 & 338 \\
\hline
\end{tabular}

Fonte: Sistema de Monitoramento de Casos de Malária na Amazônia Brasileira (SIMAM).

Tabela 2

Distribuição dos municípios com epidemias de malária, conforme a duração dos eventos epidêmicos. Amazônia Brasileira, 2010.

\begin{tabular}{|c|c|c|c|c|c|c|c|}
\hline \multirow[t]{3}{*}{ Estado } & \multicolumn{6}{|c|}{ Municípios epidêmicos conforme duração das epidemias } & \multirow[t]{3}{*}{ Tota } \\
\hline & \multicolumn{2}{|c|}{ Curta duração } & \multicolumn{2}{|c|}{ Média duração } & \multicolumn{2}{|c|}{ Longa duração } & \\
\hline & n & $\%$ & $\mathbf{n}$ & $\%$ & $\mathbf{n}$ & $\%$ & \\
\hline Acre & 6 & 42,9 & 5 & 35,7 & 3 & 21,4 & 14 \\
\hline Amazônia & 18 & 47,4 & 9 & 23,7 & 11 & 28,9 & 38 \\
\hline Amapá & 10 & 66,7 & 3 & 20,0 & 2 & 13,3 & 15 \\
\hline Maranhão & 53 & 72,6 & 17 & 23,3 & 3 & 4,1 & 73 \\
\hline Mato Grosso & 29 & 78,4 & 5 & 13,5 & 3 & 8,1 & 37 \\
\hline Pará & 54 & 48,6 & 27 & 24,3 & 30 & 27,1 & 111 \\
\hline Rondônia & 20 & 64,5 & 9 & 29,0 & 2 & 6,5 & 31 \\
\hline Roraima & 3 & 20,0 & 7 & 46,7 & 5 & 33,3 & 15 \\
\hline Tocantins & 4 & 100,0 & 0 & 0,0 & 0 & 0,0 & 4 \\
\hline Total & 197 & 58,3 & 82 & 24,3 & 59 & 17,4 & 338 \\
\hline
\end{tabular}

Fonte: Sistema de Monitoramento de Casos de Malária na Amazônia Brasileira (SIMAM).

nessas áreas, sendo responsáveis por 244.119 casos de malária (73,2\% do total da região); 111 municípios também tiveram transmissão de malária, porém fora das áreas especiais, respondendo por 5.517 casos (1,7\% do total da região). Os outros 148 municípios com áreas especiais não registraram casos autóctones da doença.

Tendo como referência a ocorrência de epidemias em 41,9\% do total de municípios da Amazônia Brasileira, esses municípios com áreas de populações mais vulneráveis de fato apresentaram maior ocorrência da situação epidêmica para a malária. A análise geral dos 807 municípios da região mostrou que, dos 477 municípios contendo áreas com populações de características especiais, 243 registraram epidemias de malária. Nos 330 municípios sem essas áreas especiais, outros 95 também registraram epidemias. Este fato mostrou que os municípios contendo áreas de populações com características especiais tiveram maior freqüência de epidemias que aqueles sem essas populações ( $\chi^{2}=39,34$; valor de $\left.\mathrm{p}<0,01\right)$.

A estratificação das áreas de populações com características especiais detectou 144 municípios com transmissão de malária em áreas de assentamentos, $75 \%$ dos quais apresentaram epidemias. A transmissão da doença nas áreas de garimpos ocorreu em 39 municípios; 79,5\% destas apresentaram epidemias. Foram identificadas 120 municípios com transmissão da doença em áreas indígenas, entre os quais 78,3\% registraram epidemias. Dos 52 municípios de fronteiras, em $82,7 \%$ foram observadas epidemias. Verificou-se que os municípios com somente áreas de assentamento tiveram maior frequência de epidemias de malária do que os municípios com áreas de 


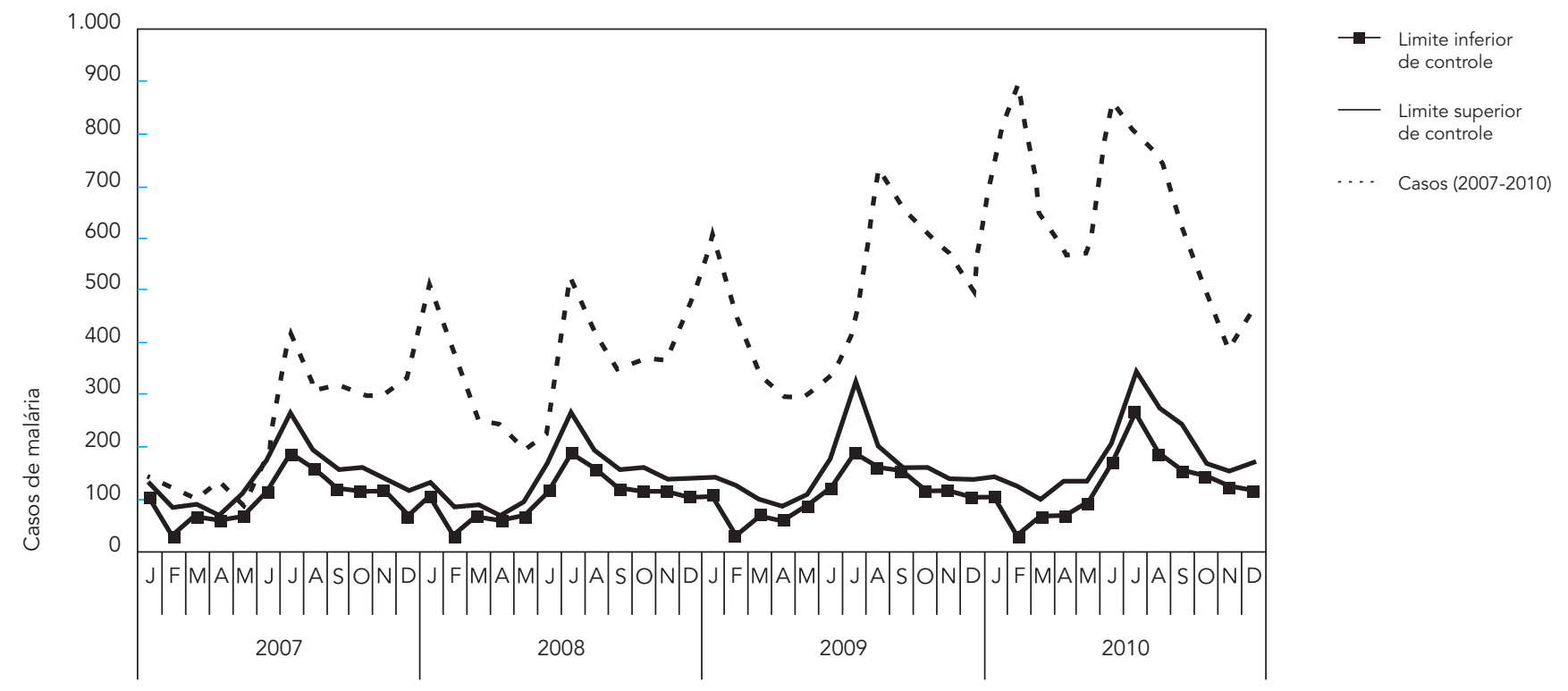

populações com características especiais $\left(\chi^{2}=\right.$ 29,24; valor de $\mathrm{p}<0,01$ ). Todavia, nos municípios com somente áreas indígenas ou somente áreas de garimpos ou de fronteiras, esse risco não foi detectado. Foram observados nove municípios com os quatro tipos de áreas especiais, os quais tiveram maior frequência de epidemias de malária do que aqueles com um, dois, ou três tipos de áreas especiais $\left(\chi^{2}=9,00\right.$; valor de $\left.p<0,01\right)$. Nos municípios onde coexistiram os quatro tipos de áreas de populações com características especiais, oito tiveram epidemias e um (Nova Mamoré - Rondônia) não registrou este fenômeno. Por outro lado, em Amajari (Roraima), Alto Alegre (Roraima) e Oriximiná (Pará), as epidemias foram de longa duração. Os demais municípios, Japurá (Amazonas), Caracarai (Rondônia), Almeirim (Pará), Vila Bela da Santíssima Trindade (Mato Grosso), registraram epidemias de curta duração, incluindo Porto Velho (Rondônia), que se destacou neste grupo por apresentar alta incidência de malária, com 23.433 casos, apesar de ter registrado epidemia de curta duração.

\section{Discussão}

Neste estudo, ficou evidenciado que quase a metade dos municípios da Amazônia Brasileira apresentou algum tipo de epidemia de malária em 2010; evidenciou-se, ainda, que os fenômenos epidêmicos ocorreram mais naqueles municípios com populações indígenas, assentamentos, garimpos e fronteiriços. Além disso, as epidemias de curta duração, entre um a quatro meses, foram mais frequentes, apesar de alguns municípios apresentarem surtos de longa duração, com mais de 40 meses. As epidemias foram produzidas tanto pelo P. falciparum quanto pelo P. vivax, separadamente, e também por ambas as espécies. Constatou-se a importância do uso automatizado da ferramenta diagrama de controle para vigilância da malária subsidiando a tomada de decisão, em tempo real, nas três esferas de gestão do Sistema Único de Saúde, a fim de reduzir a ocorrência da doença para níveis aceitáveis.

A incidência de malária pode apresentar sazonalidade relacionada à variação do clima 14 , e o aumento brusco e temporário da incidência, acima do limite superior de controle, pode ser o prenúncio da disseminação da doença 15 . Os fatores causais das epidemias são complexos e variados, envolvendo movimento populacional de suscetíveis, aumento da população dos mosquitos vetores e, também, resistência dos parasitos aos antimaláricos 9 . Portanto, a declaração técnico-operacional da epidemia deve ser reconhecida. 
O método adotado neste estudo permitiu estabelecer, com alta probabilidade estatística, o início da ocorrência epidêmica. A originalidade do SIMAM está na possibilidade de inserir uma ferramenta automatizada na rotina da vigilância da doença, de modo que os gestores municipais, estaduais e federal possam monitorar, em tempo real, a incidência da malária (Figura 1). Embora este método apresente alta sensibilidade, suficiente para identificação das epidemias 10 , a especificidade em $75 \%$ do terceiro quartil poderá, eventualmente, disparar alarmes falsos positivos, os quais, em princípio, não trarão prejuízos para o controle da malária, por demandarem maior precocidade no início das ações de contenção do fenômeno epidêmico.

A utilização do número de casos para construção do limite superior do diagrama de controle converge com a definição de epidemia adotada pela OMS 12 e por outros autores, como Medronho \& Perez 13 e Pereira 16. O número absoluto de casos é recomendado também pela OMS 9 para detecção de epidemias de malária, tendo sido utilizado em países como Tailândia 5, Quênia 14 e Peru 17. Diferentemente, na detecção de um foco de malária na República da Zâmbia 18, foi utilizada a taxa de incidência parasitária, porém acredita-se que essa taxa apresente limitações em relação ao caráter focal da endemia, pois reduz a sensibilidade do diagrama de controle, tornando tardia a detecção das epidemias em áreas com grandes populações e vastas extensões territoriais. Além disso, dificulta a detecção da reintrodução da doença em municípios livres de transmissão, onde a presença de poucos casos implica taxa de incidência igual a zero.

A série de dados mensais utilizada para o cálculo do limite superior do diagrama de controle está de acordo com o que preconizam a OMS 9, Medronho \& Perez 13 e Pereira 16. Esse procedimento também foi adotado por Cullen et al. 5, os quais, com base em uma série histórica de sete anos, excluíram dois anos com maior número de casos; o mesmo critério foi utilizado ainda por Braz et al. ${ }^{10}$. Por sua vez, Hay et al. ${ }^{14}$ não excluíram os anos epidêmicos, adotando, simplesmente, os cinco anos anteriores ao ano de monitoramento. Em virtude da dinâmica da malária, que apresenta grande variabilidade de um ano para o outro e característica variação sazonal esperada, como ocorre na maioria dos municípios da Amazônia Brasileira, considerou-se importante manter o conjunto de sete anos retrospectivos, com exclusão dos dois meses com maior número de casos, em cada ano, para melhorar o poder de mensuração da estimativa.

Em face da complexidade dos fatores envolvidos no surgimento das epidemias, é necessário utilizar metodologias objetivas, que permitam detectar rapidamente o fenômeno, mesmo sem considerar, inicialmente, todas as causas ${ }^{19}$, conforme ocorreu neste estudo. Verifica-se, assim, a necessidade de intervenção imediata, com diagnóstico da doença e tratamento oportunos das pessoas, além da busca dos fatores causais para contenção do avanço da epidemia. Esta, uma vez iniciada, requer investigação para estabelecer o local exato, a extensão do surto, as espécies envolvidas, as causas e medidas de controle apropriadas. É importante que o sistema de vigilância tenha sensibilidade suficiente para detectar quaisquer situações fora dos padrões, permitindo respostas rápidas na ocorrência de incidências anormais.

\section{Epidemias por espécie de Plasmodium}

Existem, no Brasil, três espécies de Plasmodium (P.falciparum, P.vivaxe P. malariae), que resultam em diferentes doenças em populações e locais distintos 20. Foi possível verificar que, a despeito de alguns municípios apresentarem epidemias individualizadas por espécie, na Amazônia Brasileira, as epidemias são produzidas pelos $P$. vivax e $P$ falciparum, estando de acordo com o que se encontra em outros países fora da África, onde as epidemias, geralmente, devem-se à superposição das duas espécies parasitárias 15 (Tabela 1). O surgimento de epidemias por P. falciparum, apesar dos fatores socioambientais, demonstra possíveis falhas nas ações de controle, pois, quando se detecta epidemia por esta espécie, é porque houve retardo no diagnóstico do caso índice. Os gametócitos de P. falciparum, formas infectantes para os mosquitos vetores, surgem no sangue periférico de sete a dez dias após a invasão pelos trofozoítas, enquanto no $P$. vivax aparecem simultaneamente aos trofozoítas 15 . Os três agrupamentos de municípios por tipos de epidemias (Tabela 1) mostraram a necessidade do monitoramento rotineiro por espécie parasitária, separadamente. Os municípios com epidemias dos tipos I e II evidenciaram que, antes do aparecimento do surto produzido pelo conjunto das duas espécies (epidemia tipo III), pode ocultar-se outra epidemia silenciosa, produzida pelo P. falciparum ou pelo P. vivax.

\section{Duração das epidemias}

Na Amazônia Brasileira, em locais onde convivem as duas espécies parasitárias, os casos de $P$. vivax são atualmente mais abundantes, seguidos de P.falciparum, cujas epidemias geralmente são mais duradouras, conforme observado neste estudo. A variabilidade na duração das 
epidemias, de um mês a mais de três anos, está relacionada a diferentes fatores, como o desmatamento de áreas extensas, grandes migrações, formação de aglomerações desprotegidas e a falta de infraestrutura 21 .

O prazo entre um e quatro meses é considerado adequado para detecção e contenção das epidemias de malária, uma vez que a maioria dos municípios conseguiu controlar os eventos nesse intervalo de tempo. Porém, o ideal é que a epidemia seja detectada e controlada o mais rápido possível ${ }^{9}$. A duração superior a esse prazo pode se dever às falhas nas ações de controle ou deficiências na estrutura dos serviços de saúde, conforme verificado nos municípios Espigão D’Oeste, Santa Cruz do Arari e Goianésia do Pará; nessas localidades, as epidemias se prolongaram por vários anos, embora pudessem ter sido detectadas e contidas oportunamente com auxílio de ferramenta idêntica ao SIMAM.

Estudo realizado no Irã usou a duração mínima de quatro semanas para medir a sensibilidade, especificidade e oportunidade dos alertas, permitindo a melhor definição das epidemias 22, no entanto as séries históricas analisadas no presente estudo não permitiram esse controle semanal. Mesmo assim, o SIMAM detectou desde epidemias de apenas um caso da doença e com duração de um mês, até aquelas com duração de mais de três anos e centenas de casos.

\section{Epidemias em municípios com áreas de populações com características especiais}

Nesta pesquisa, observou-se que a ocorrência de epidemias em municípios com áreas de populações de características especiais (indígenas, assentamentos, garimpos e municípios de fronteiras) de fato foi superior à média das epidemias verificadas no total de municípios dos estados da Amazônia Brasileira. Os elevados percentuais de epidemias em municípios com áreas indígenas, de assentamentos, de garimpos e de fronteiras evidenciaram o agravamento da malária nesses locais, tornando adequada a classificação dessas áreas como de populações com características especiais, pois geralmente são áreas com a presença de pessoas não imunes, vivendo em condições precárias de habitação e trabalho. Esse quadro facilita a transmissão da doença, em face do aumento da exposição e consequentemente do contato vetor/habitantes, e requer maior atenção dos serviços de saúde, já que, conforme foi constatado, nos municípios com essas áreas especiais, o risco de apresentar epidemias de malária é maior do que nos municípios sem essas áreas. Ademais, quanto mais tipos de áreas especiais coexistirem no município, maior também será o risco de ocorrerem epidemias de malária ocasionadas pela complexidade das atividades desenvolvidas nessas localidades.

Atividades garimpeiras dificultam as intervenções de controle, uma vez que não possuem organização profissional. Em adição, os trabalhadores vivem em áreas de difícil acesso, em ambiente que favorece a proliferação do vetor, pois o acúmulo de água nas cavas abandonadas leva ao aumento do número de criadouros. Estudo realizado no Estado de Mato Grosso detectou forte associação entre atividade garimpeira e aumento na incidência da malária 23 . Outro estudo atribuiu o incremento de casos de malária à instalação de garimpos num projeto de assentamento 24. Por outro lado, em exploração mineral regularizada, a participação da iniciativa privada no controle da malária mostrou-se importante para a redução da incidência da doença 25 .

Nas áreas de assentamentos, a transmissão é alta por causa da derrubada de matas para plantio e pela presença de população suscetível vinda de áreas sem transmissão 26. No Município de Juruena (Mato Grosso), os habitantes de um assentamento, provenientes de áreas não endêmicas, tiveram probabilidade de infecção 2,9 vezes maior do que aqueles vindos de áreas endêmicas, possivelmente pela baixa imunidade e falta de conhecimento sobre medidas de proteção 27 . Geralmente, nos anos iniciais, o assentamento apresenta alto risco para epidemias, determinado pelas transformações ambientais. Após dez anos, o perfil de alto risco passa a ser caracterizado pelos aspectos econômicos e comportamentais 28 . Estes fatores reforçam os achados do presente estudo, que demonstrou que os municípios com somente áreas de assentamentos tiveram maior risco de apresentar epidemias de malária do que os municípios com as demais áreas especiais.

Deve-se ressaltar que o risco de contrair a doença na população indígena ainda é duas vezes maior do que na população da Amazônia Brasileira 29, corroborando o alto percentual de epidemias encontrado nos municípios com áreas indígenas. Porém, com a organização dos serviços de saúde, a ampliação da rede de diagnóstico e tratamento, além do comprometimento intersetorial no controle da malária, os indicadores da doença nessas áreas podem ser melhorados, conforme verificado em estudo sobre o controle da malária em um município amazônico, onde mais de $90 \%$ da população é indígena 30 .

A transmissão da malária nas áreas de fronteira não só é pouco estudada, apesar de haver muitas iniciativas para controle integrado da doença entre o Brasil e outros países, como também é influenciada pela diversidade e complexidade das relações que se estabelecem no espaço fron- 
teiriço ${ }^{31}$. No mais alto grau de interação fronteiriça, os municípios gêmeos são condicionantes para mobilidade que favorece a transmissão da malária, como ocorre nas fronteiras Oiapoque (Amapá)-Saint-Georges (Guiana Francesa), Pacaraima (Roraima)-Santa Elena de Uairén (Venezuela) e Bonfim (Roraima)-Lethem (Guiana). Nessas áreas, a atenção à saúde é marcada pela carência de profissionais qualificados, principalmente médicos e enfermeiros, com grandes dificuldades de acesso das populações aos serviços de saúde, exceto nas capitais dos estados. A elevada mobilidade transfronteiriça dos indígenas, a dificuldade de acesso à região pelas equipes de saúde e a persistente incursão de garimpeiros dificultam as ações de controle da malária. Outros fatores que contribuem para o avanço da doença é o processo migratório, com desmatamento de longas áreas para ocupação e o contato entre madeireiros, garimpeiros e povos indígenas 32 . Esse

\section{Resumen}

Las epidemias de malaria ocurren anualmente en los municipios de la Región Amazónica, Brasil, no obstante, los servicios de salud no adoptan de manera sistemática instrumentos para la detección y contención oportuna de este tipo de eventos. El objetivo fue caracterizar las epidemias de malaria en la región según su duración, especie de Plasmodium y vulnerabilidad de las poblaciones. Se evalúo un sistema de supervisión automatizado de la incidencia de la malaria, en base al diagrama de control según cuartiles, con el fin de identificar las epidemias de la enfermedad. En 2010, se produjeron epidemias en 338 (41,9\%) municipios de la región. Hubo epidemias por $\mathrm{P}$. falciparum y por P. vivax, separadamente, y también por ambas especies. Hubo epidemias con una duración de uno a cuatro meses que se produjeron en un 58,3\% de los municipios epidémicos; de cinco a ocho meses, en un 24,3\%; $y$ de nueve a 12 meses, en un 17,4\%. La supervisión automatizada de la variación de la incidencia de la malaria podrá contribuir a la detección precoz de las epidemias y mejorar su control adecuado.

Malaria; Plasmodium; Epidemia processo de transmissão da malária nas áreas de fronteira internacional foi verificado em outros países, nos quais a mobilidade de garimpeiros provocou o surgimentodeepidemias dadoença ${ }^{33}$.

O extrativismo vegetal de produtos como castanhas, palmitos e açaí tem contribuído para as epidemias de malária na região, em virtude da grande exposição dos trabalhadores às picadas dos mosquitos vetores da doença, dentro da mata. Outros fatores, como o desflorestamento de grandes áreas, estão associados à alta incidência da malária 34. Por saturação do lençol freático, os reservatórios das usinas hidrelétricas aumentam o número e a perenidade dos criadouros. É importante que os serviços de saúde superem a etapa de detecção e contenção das epidemias com metas mais ambiciosas, voltadas para redução sustentável e, até mesmo, eliminação da doença em algumas áreas com baixa incidência de casos.

\section{Colaboradores}

R. M. Braz, E. C. Duarte e P. L. Tauil participaram da concepção, projeto, análise e interpretação dos dados, além da redação do artigo e aprovação final da versão a ser publicada. 


\section{Referências}

1. World Health Organization. World malaria report 2010. http://www.who.int/malaria/world_ma laria_report_2010/worldmalariareport2010.pdf (acessado em 30/Out/2011).

2. Loiola CCP, Silva CJM, Tauil PL. Controle da malária no Brasil: 1965 a 2001. Rev Panam Salud Pública $2002 ; 11: 235-44$.

3. Oliveira-Ferreira J, Lacerda MV, Brasil P, Ladislau JL, Tauil PL, Daniel-Ribeiro CT. Malaria in Brazil: an overview. Malar J 2010; 9:115.

4. Hay SI, Were EC, Renshaw M, Noor AM, Ochola SA, Olusanmi I, et al. Forecasting, warning, and detection of malaria epidemics: a case study. Lancet 2003; 361:1705-6.

5. Cullen JR, Chitprarop U, Doberstyn EB, Sombatwattanangkul K. An epidemiological early warning system for malaria control in the northern Thailand. Bull World Health Organ 1984; 62:107-14

6. Abeku TA. Response to malaria epidemics in Africa. Emerg Infect Dis 2007; 13:681-6.

7. Confalonieri UEC. Saúde na Amazônia: um modelo conceitual para a análise de paisagens e doenças. Estud Av 2005; 19:221-36.

8. Barbieri AF. Uso do solo e prevalência de malária em uma região da Amazônia Brasileira. Cadernos de Geografia 2005; 15:9-30.

9. World Health Organization. Field guide for malaria epidemic assessment and reporting. Geneva: World Health Organization; 2004.

10. Braz RM, Andreozzi VL, Kale PL. Detecção precoce de epidemias de malária no Brasil: uma proposta de automação. Epidemiol Serv Saúde 2006; 15:21-33.

11. Braz RM, Duarte EC, Tauil PL. Epidemiology of malaria in the municipality of Cruzeiro do Sul State of Acre Brazil in 2010: uses of a control chart at the local level. Rev Soc Bras Med Trop 2012; 45:526-9.

12. World Health Organization. Disease outbreaks. Geneva: World Health Organization; 2011.

13. Medronho RA, Perez MA. Distribuição das doenças no espaço e no tempo. In: Medronho RA, Carvalho DM, Bloch KV, Luiz RR, Werneck GL, organizadores. Epidemiologia. São Paulo: Editora Atheneu; 2003. p. 57-71.

14. Hay SI, Simba M, Busolo M, Noor AM, Guyatt HL, Ochola SA, et al. Defining and detecting malaria epidemics in the highlands of western Kenya. Emerg Infect Dis 2002; 8:555-62.

15. World Health Organization. Malaria epidemics detection and control forecasting and prevention. Geneva: World Health Organization; 1998.

16. Pereira MG. Epidemiologia: teoria e prática. Rio de Janeiro: Editora Guanabara Koogan; 1995.

17. Calderon L, Valencia W, Albujar J. Análisis de la epidemia de malaria en la zona del Alto Pastaza - región Loreto (1989-1996). Rev Peru Epidemiol 1996; 9:19-23.

18. Davis RG, Kamanga A, Castillo-Salgado C, Chime N, Mharakurwa S, Shiff C. Early detection of malaria foci for targeted interventions in endemic southern Zambia. Malar J 2011; 10:260.

19. Rodríguez J, Prieto S. Dinámica de la epidemia de malaria: predicciones de su trayectoria. Rev Med 2010; 18:152-60.
20. Alonso PL, Brown G, Arevalo-Herrera M, Binka F, Chitnis C, Collins F, et al. A research agenda to underpin malaria eradication. PLoS Med 2011; 8:e1000406.

21. Vasconcelos CH, Novo EMLM, Donalisio MR. Uso do sensoriamento remoto para estudar a influência de alterações ambientais na distribuição da malária na Amazônia Brasileira. Cad Saúde Pública 2006; 22:517-26

22. McKelvie WR, Haghdoost AA, Raeisi A. Defining and detecting malaria epidemics in south-east Iran. Malar J 2012; 11:81.

23. Duarte EC, Fontes CJF. Associação entre produção anual de ouro em garimpos e incidência de ma lária em Mato Grosso. Brasil, 1985-1996. Rev Soc Bras Med Trop 2002; 35:665-8.

24. Santos VR, Yokoo EM, Souza-Santos R, Atanaka Santos M. Fatores socioambientais associados à distribuição espacial de malária no assentamento Vale do Amanhecer, Município de Juruena, Estado de Mato Grosso, 2005. Rev Soc Bras Med Trop 2009; 42:47-53.

25. Couto AA, Calvosa VS, Lacerda R, Castro F, Rosa ES, Nascimento JM. Controle da transmissão da malária em área de garimpo no Estado do Amapá com participação da iniciativa privada. Cad Saúde Pública 2001; 17:897-907.

26. Barata RCB. Malária no Brasil: panorama epidemiológico na última década. Cad Saúde Pública 1995; 11:128-36.

27. Oliveira EC, Santos ES, Zeilhofer P, Souza-Santos R, Atanaka-Santos M. Spatial patterns of malaria in a land reform colonization project, Juruena municipality, Mato Grosso, Brazil. Malar J 2011; 10:177.

28. Castro MC, Singer BH. Meio ambiente e saúde: metodologia para análise espacial da ocorrência de malária em projetos de assentamento. Rev Bras Estud Popul 2007; 24:247-62.

29. Secretaria de Vigilância em Saúde, Ministério da Saúde. Programa Nacional de Controle da Malária. Brasília: Ministério da Saúde; 2004.

30. Rodrigues EC, Lopes Neto D. Malaria control in an Amazon municipality. Rev Latinoam Enferm 2011; 19:1297-305.

31. Grupo de Trabalho Interfederativo de Integração Fronteiriço, Ministério da Integração Regional. Bases para uma proposta de desenvolvimento e integração da faixa de fronteira. Brasília: Ministério da Integração Regional; 2010.

32. Peiter PC. A geografia da saúde na faixa de fronteira continental do Brasil na passagem do milênio [Tese de Doutorado]. Rio de Janeiro: Universidade Federal do Rio de Janeiro; 2005.

33. Konchom S, Singhasivanon P, Kaewkungwal J, Chuprapawan S, Thimasarn K, Kidson C, et al. Chronicle of malaria epidemics in Thailand, 1980-2000. Southeast Asian J Trop Med Public Health 2005; 36 Suppl 4:64-7.

34. Olson SH, Gangnon R, Silveira GA, Patz JA. Deforestation and malaria in Mâncio Lima County, Brazil. Emerg Infect Dis 2010; 16:1108-15.

Recebido em 22/Ago/2012

Versão final reapresentada em 12/Nov/2012

Aprovado em 08/Jan/2013 